\title{
Tuning Molecular Vibrational Energy Flow within an Aromatic Scaffold via Anharmonic Coupling
}

\author{
Andrew J. Schmitz ${ }^{a}$, Hari Datt Pandey ${ }^{c}$, Farzaneh Chalyavi ${ }^{\mathrm{a}}$, Tianjiao Shi ${ }^{\mathrm{b}}$, Edward E. \\ Fenlon $^{b}$, Scott H. Brewer ${ }^{b}$, David M. Leitnera ${ }^{a}$, Matthew J. Tucker ${ }^{\star}$,a \\ aDepartment of Chemistry, University of Nevada, Reno, Nevada 89557, United States \\ bDepartment of Chemistry, Franklin \& Marshall College, Lancaster, Pennsylvania 17604-3003, \\ United States \\ 'Department of Chemistry, University of California Riverside, California, 92521, United States
}

\section{Abstract}

\begin{abstract}
From guiding chemical reactivity in synthesis or protein folding to the design of energy diodes, intramolecular vibrational energy redistribution harnesses the power to influence the underlying fundamental principles of chemistry. To evaluate the ability to steer these processes, the mechanism and timescales of intramolecular vibrational energy redistribution through aromatic molecular scaffolds have been assessed by utilizing two-dimensional infrared (2D IR) spectroscopy. 2D IR cross peaks reveal energy relaxation through an aromatic scaffold from the azido- to the cyano- vibrational reporters in para-azidobenzonitrile (PAB) and para(azidomethyl)benzonitrile (PAMB) prior to energy relaxation into the solvent. The rates of energy transfer are modulated by Fermi resonances, which are apparent by the coupling cross peaks identified within the 2D IR spectrum. Theoretical vibrational mode analysis allowed determination of the origins of the energy flow, the transfer pathway, and a direct comparison of the associated transfer rates, which were in good agreement with the experimental results. Large variations in energy transfer rates, approximately 1.9 ps for PAB and 23 ps for PAMB, illustrate the importance of strong anharmonic coupling, i.e. Fermi resonance, on the transfer pathways. In particular, vibrational energy rectification is altered by Fermi resonances of the cyano- and azido- modes allowing control of the propensity for energy flow.
\end{abstract}

\section{Keywords}

Vibrational Energy Flow; 2D IR; Anharmonic Coupling; Probe Pairs; Fermi Resonance

\footnotetext{
*Corresponding Author: Dr. Matthew J. Tucker, mtucker@unr.edu. Author Contributions

MJT, DML, EEF and SHB designed the research. AJS and FC performed the laser experiments. AJS and MJT provided expertise in setup and optical design. HDP and DML calculated the theoretical data. TS and EEF synthesized the model systems; TS and SHB measured preliminary IR data; AJS, FC, HDP, DML, and MJT analyzed the data; AJS, FC, HDP, EEF, DML, and MJT wrote the paper. All authors revised and edited the manuscript.

ASSOCIATED CONTENT

Details of the synthesis, theoretical data, the cyano- to the azido- cross peak analysis, analysis on the bi-exponential fit of the cross peaks, derivation of the kinetic scheme, and dipolar coupling can be obtained in the Supporting Information. This material is available free of charge via the Internet at http://pubs.acs.org.
} 


\section{Introduction}

For the past two decades two-dimensional infrared (2D IR) spectroscopy has become the premiere ultrafast technique to characterize vibrational dynamics. It has been used to study a wide array of systems, such as structural dynamics in biomolecules, energy transfer in materials, population exchange, and properties of ion channels in cell membranes. ${ }^{1-7}$ Additionally, the localized solvent dynamics in heterogeneous solutions, which play an important role in reactivity, are also determined through spectral diffusion observed using 2D IR spectroscopy. ${ }^{8-12}$ Another valuable characteristic of 2D IR is the ability to detect interactions between multiple vibrational transitions within a molecular system. For example, the presence of vibrational coupling, i.e. mechanical and/or dipole-dipole coupling $^{5,13-14}$, is oftentimes monitored through the presence of cross peaks in the 2D IR spectrum at the earliest evolution or waiting times. ${ }^{151,5,16-18}$ Cross peaks also can appear as a function of later waiting times. These spectral features measure the dynamics resulting from vibrational energy relaxation (VER). In this case, the two modes are delocalized within a molecular system causing thermal energy flow via VER within the molecule as intramolecular vibrational energy redistribution (IVR) or through the solvent as intermolecular vibrational energy transfer. ${ }^{19-23}$ The overall process is delayed since the energy absorbed from one detected mode must propagate within the molecule until reaching the other detected mode, reflected by the intensity growth timescale. ${ }^{19}, 21,24$ VER have been studied both theoretically ${ }^{25-34}$ and experimentally, where nonlinear techniques, such as IR pump Raman probe and 2D IR, have provided great insights into the rates of VER, direction of heat flow, and diffusive/ballistic transport. ${ }^{19,} 21,35-42$ Despite the vast knowledge acquired from such studies, the overall process of VER still remains challenging to understand. The ambiguity in VER, in particular IVR, arises because the energy transfer occurs from one bright mode to another mode via multiple dark states. This process creates difficulties in determining the rate and exact pathway of energy transfer within the molecular system. ${ }^{22}$ Since intramolecular pathways are often more favorable, there are limitations in how much energy will propagate through the solvent. This complicates intermolecular energy transfer studies. However, Zheng and coworkers have developed methods to design molecules that decouple the modes making intermolecular vibrational energy transfer the preferred route. ${ }^{22,43}$

VER plays a vital role in many physical and chemical processes, such as chemical reactions where selected vibrational energy flow influences the reaction coordinate aiding in synthetic chemistry, ${ }^{20}, 22,40,43-46$ molecular electronics and electrochemistry, ${ }^{38-39,47-49}$ measuring three dimensional structures of molecular systems, ${ }^{24,}, 50-53$ and energetic properties of transition metals. ${ }^{20-21,41,48}$ VER also influences protein functions through hydrogen bonding along protein backbones, ${ }^{32,}, 54$ formation of secondary and tertiary structures, ${ }^{34}$ and allosteric communication. ${ }^{55-56}$ By learning ways to control VER, the ability to influence these processes in a desirable way becomes possible.

Several recent 2D IR studies have expanded our understanding of VER and some of the factors guiding its pathway. The strong carbonyl mode in transition metal complexes was used by Kubarych and co-workers to investigate the effects of water on the relaxation of the carbonyl. ${ }^{57}$ The IVR rate of ruthenium versus iron complexes were compared, ${ }^{21}$ and the 
influence of hydrogen bonding on IVR rates was determined. ${ }^{58}$ Other studies have captured the energy transfer through transition metals suggesting a heavy atom can influence efficiency, direction of IVR, and the contribution of solvent on IVR. ${ }^{41,59-61}$ Zheng and coworkers have contributed an abundance of work on intermolecular energy transfer via 2D IR, including the determination of energy transfer rates, distances, and orientations between two modes on different molecules..$^{22,40,43,47,49-51}$ Rubtsov and co-workers have established methods of coupling involving IVR coined relaxation-assisted two-dimensional infrared spectroscopy (RA 2DIR). ${ }^{19}$ Rubtsov developed his methodology based on observations of IVR between a variety of vibrational mode pairs, ${ }^{24,} 62-6465$ including $\mathrm{CN} / \mathrm{CO}, \mathrm{CN} /$ amide I modes, and $\mathrm{N}_{3} / \mathrm{CO}$, in several molecular scaffolds with varying configurations and bond distances between the transitions. His work has uncovered a correlation between energy transfer rate and bond length, ${ }^{49,53,63}$ temperature dependence of energy transfer rates, ${ }^{65}$ and cross peak enhancement. ${ }^{48,62-63}$

Our group, as shown in previous work, has an interest in studying the vibrational dynamics of the azido- $\left(\mathrm{N}_{3}\right)$ and cyano- $(\mathrm{CN})$ transitions within biological systems. ${ }^{4-5,66}$ These modes have been established as valuable infrared probes due to their sensitivity to their local environment, small size to limit perturbation to the molecular system, and the large transition dipole strength of azides, which increases the signal-to-noise ratio. Lastly, in biological systems, these transitions are spectrally isolated from other congested regions of the IR spectrum. ${ }^{4-5,67-68}$ Recently, these transitions have been placed in tandem on molecular systems revealing dipolar coupling for structural studies within different biological scaffolds. ${ }^{4-5}$ To further examine the coupling mechanisms of these probe pairs, several molecular models were designed and synthesized, including para-azidobenzonitrile (PAB) and para-(azidomethyl)benzonitrile (PAMB). Within these testing manifolds, our 2D IR measurements detected the presence of some dipole-dipole coupling and extensive IVR. Herein, the details of the coupling processes for these systems are determined through a combination of experimental and theoretical methods showing that the molecular energy flow is tuneable within this aromatic scaffold via alteration of the anharmonic mode coupling. Our approach uses a model system to uncover the coupling manifold between the azido- transition, several combination bands, and the cyano- transition to illustrate how the transfer pathway can be influenced to guide the energy flow. This information is necessary for the design of molecular electronics. Furthermore, by limiting the IVR pathway to one direction, molecular selectivity in synthesis and biological function may become possible.

\section{Experimental}

\section{Synthesis of PAB and PAMB}

4-azidobenzonitrile was prepared by a literature method. ${ }^{69-70}$ Complete synthetic details are given in the Supporting Information. In short, 4-aminobenzonitrile was diazotized with nitrous acid and then treated with sodium azide to give PAB in $85 \%$ yield. 4(azidomethyl)benzonitrile was prepared by the SN2 reaction of sodium azide with 4bromomethyl benzonitrile to provide 4-(azidomethyl)benzonitrile in $83 \%$ yield. This is essentially the method of Chakraborty et al., ${ }^{71}$ except that aqueous methanol was used as the solvent instead of DMSO. 


\section{Linear and 2D IR Measurements}

FTIR and 2D IR measurements were performed in a solution of tetrahydrofuran (THF) with a concentration of approximately $30 \mathrm{mM}$ using $\mathrm{CaF}_{2}$ windows in a Harrick sample cell with a path length of $50 \mu \mathrm{m}$. Linear IR measurements were performed using a Nicolet 6700 FTIR spectrometer.

2D IR experiments were performed using Fourier-transform limited $80 \mathrm{fs}$ pulses at a center wavelength of $4550 \mathrm{~nm}$. Three pulses of wave vectors $\mathrm{k}_{1}, \mathrm{k}_{2}$, and $\mathrm{k}_{3}$ with energy of $\sim 1 \mu \mathrm{J}$ were incident on the sample generating an echo signal in the direction $\mathrm{k}_{\mathrm{s}}=-\mathrm{k}_{1}+\mathrm{k}_{2}+\mathrm{k}_{3}$. Pulse order of 123 and 213 produced rephasing and nonrephasing, respectively. Both rephasing and nonrephasing were properly phased and combined creating the absorptive spectrum. Spectral changes over time were observed by varying the waiting time $\left(\mathrm{T}_{\mathrm{W})}\right.$ between the second and third pulse from 0 to 12 ps. Appropriate Fourier transforms along the coherence $(\tau)$ and detection (t) axes were performed and plotted as $\omega_{\tau}$ vs $\omega_{t}{ }^{5}$

\section{Computations}

The initial geometry of each compound was constructed using the Avogadro visualization package and optimized using molecular mechanics (MM) with the General Amber Force Field (GAFF). The MM optimized geometry was introduced into a semi-empirical method (PM6) optimization, followed by a Hartree-Fock level (HF) calculation with the 6-31G basis set applying the conducting polarizable continuum model (CPCM) THF model for the selfconsistent reaction field (SCRF). The HF optimized geometry was taken as an initial structure for a DFT/B3LYP/6-31G-level calculation, followed by DFT/B3LYP/6-31+G* using the same solvent model for the SCRF. Finally, the geometry, Hessian, normal modes, frequencies, and anharmonic constants were calculated using DFT/B3LYP/6-31+G**. An ultrafine integration grid for the two-electron integral calculations with accuracy 10-13 and very tight convergence criteria were applied throughout the electronic structure calculation process. All the DFT calculations were carried out using the Gaussian-09 computational package. In order to determine a stable third order coefficient, it was necessary to use the above-mentioned calculation protocol. The CPCM model for the THF solvent was utilized at each level of theory.

The rate of relaxation of excess energy in the azido- and cyano- modes is mediated by the anharmonic interactions with other modes of the molecule and interactions with the solvent. The relaxation rate $(W)$ was estimated in terms of third order anharmonic interaction with a Fermi's golden rule calculation for excess energy in mode a with frequency $\omega_{a}$, which can be expressed as the sum of quantities "decay" and "collision" terms, $W=W_{d}+W_{c}$ where $25-31,72-73$

$$
W_{d}\left(\omega_{\alpha}\right)=\frac{\hbar}{16 \omega_{\alpha}} \sum_{\beta, \gamma}\left\{\frac{\left|\phi_{\alpha \beta \gamma}\right|^{2}\left(1+n_{\beta}+n_{\gamma}\right)\left(\Gamma_{\alpha}+\Gamma_{\beta}+\Gamma_{\gamma}\right)}{\omega_{\beta} \omega_{\gamma}\left(\omega_{\beta}+\omega_{\gamma}-\omega_{\alpha}\right)^{2}+\frac{1}{4}\left(\Gamma_{\alpha}+\Gamma_{\beta}+\Gamma_{\gamma}\right)^{2}}\right\}
$$




$$
W_{c}\left(\omega_{\alpha}\right)=\frac{\hbar}{8 \omega_{\alpha}} \sum_{\beta, \gamma}\left\{\frac{\left|\phi_{\alpha \beta \gamma}\right|^{2}\left(n_{\beta}-n_{\gamma}\right)\left(\Gamma_{\alpha}+\Gamma_{\beta}+\Gamma_{\gamma}\right)}{\omega_{\beta} \omega_{\gamma}\left(\omega_{\gamma}-\omega_{\beta}-\omega_{\alpha}\right)^{2}+\frac{1}{4}\left(\Gamma_{\alpha}+\Gamma_{\beta}+\Gamma_{\gamma}\right)^{2}}\right\}
$$

$\Gamma_{\beta}$ is the damping rate of mode $\beta$ due to coupling to other modes and to the environment, $n_{a}$ is the Boltzmann population of mode $a$, and $\Phi_{a \beta \gamma}$, are cubic anharmonic constants. Since the vibrational relaxation rate that is calculated influences the value of $\Gamma$, this parameter was calculated self-consistently solving the equation $\Gamma_{a}-W_{d}-W_{c}=0$ using a NewtonRaphson scheme iteratively as detailed in Ref. 73. Resonant coupling can be characterized by the relative magnitude of the cubic anharmonic constant to the frequency mismatch of the coupled modes $(\Delta \omega)$ defined as $\left|\omega_{\gamma}+\omega_{\beta}-\omega_{a}\right| . \Delta \omega$ is also referred to as the resonance distance. The ratio of the magnitude of the anharmonic coupling to the resonance distance is the third-order Fermi resonance parameter for the triple of modes $a \beta \gamma$, defined by,

$$
\operatorname{TFR}_{\alpha \beta \gamma}=\left|\frac{\Phi_{\alpha \beta \gamma}}{\Delta \omega}\right|
$$

Modes are resonant when TFR is of order 1 or larger. The damping rates that appear in Eq. (1) have contributions from coupling to solvent. The damping rate for a vibrational mode due to solvent coupling is taken to be roughly $0.2 \mathrm{ps}^{-1}, 33,38$ and we add to the overall damping rate for the triple of modes in Eq. (1) the value $0.6 \mathrm{ps}^{-1}$ to account for solvent coupling. We note that we found in earlier work on solvated cyanophenylalanine that the results of Eq. (1) do not change significantly using a range of values from $0.2 \mathrm{ps}^{-1}$ to $1.5 \mathrm{ps}$ ${ }^{-1}$ for the contribution of coupling to the solvent. ${ }^{73}$

\section{Results and Discussion}

\section{Linear IR}

FTIR and 2D IR measurements were performed on para-(azidomethyl)benzonitrile (PAMB) and para-azidobenzonitrile (PAB) in THF. Four transitions are observed in the linear IR spectrum for PAMB and PAB as shown in Figure 1A and 1B, respectively. For the PAB compound, the asymmetric stretch of the azido- transition appears at $2106 \mathrm{~cm}^{-1}$ and the cyano- stretch is located at $2228 \mathrm{~cm}^{-1}$. Another transition at $2138 \mathrm{~cm}^{-1}$ arises from a Fermi resonance that has been reported with the azido- reporter in other ring systems in various solvents. ${ }^{67,74-76}$ The origin of this transition is further supported by anharmonic frequency computations suggesting only two transitions within this spectral region. A fourth transition is observed at $2200 \mathrm{~cm}^{-1}$ resulting from an intrinsically weak solvent band of THF, ${ }^{77}$ and thus it is disregarded from further analysis. For the PAMB compound, the IR spectrum is quite similar to that of PAB with only a change in the relative intensity of the spectral features. The azido- and cyano- transitions are located at $2103 \mathrm{~cm}^{-1}$ and $2230 \mathrm{~cm}^{-1}$, respectively. In the spectral profile of PAMB, the transition observed at $2130 \mathrm{~cm}^{-1}$ is likely due to the aforementioned Fermi resonance. However, the intensity of this vibrational transition is much weaker when compared to the Fermi transition of PAB. The single carbon atom separation between the azido- group and the benzene ring is likely decoupling the ring modes of benzene and the azido- transition. ${ }^{78}$ 
The experimental relative IR intensity ratio between the azido- to cyano- stretches was determined to be $3.4: 1$ and 4.7:1 for PAMB and PAB, respectively. The observed trend in the intensity ratios is in agreement with with the DFT calculated trend for PAMB (5.9:1) and PAB (8.8:1). Discrepancies in the absolute intensity ratios are in part due to the effect of the intensity mixing caused by the Fermi resonance coupling within the experiment.

Theoretical calculations predict multiple transitions that possibly gives rise to the Fermi resonance present in the linear IR spectrum. The most probable ones are listed in Table 1 and shown in Figure 2 (see SI for more details). The overall coupling strength increases when the magnitude of the third order coupling constant, $\Phi_{\alpha \beta \gamma}$, increases and/or when a decrease in the resonance distance, $\Delta \omega$, is observed resulting in a larger value of the third order resonance parameter, $\mathrm{TFR}_{\alpha \beta \gamma}$. Typically, if $\mathrm{TFR}_{\alpha \beta \gamma} \geq 1, \Phi_{\alpha \beta \gamma}>10 \mathrm{~cm}^{-1}$ and $\Delta \omega<10$ $\mathrm{cm}^{-1}$ then the overall coupling strength will be significant enough to result in an accidental resonance between the combination bands and the fundamental transition, i.e. a Fermi resonance.. Based on these criteria, it is clear to see from Table 1 that the TFR is largest ( 1.9) between the azido- mode and a combination band suggesting these modes contribute to the Fermi resonance in PAB and further substantiate the other strong transitions observed in the IR spectrum due to anharmonic coupling and intensity mixing. On the other hand, the TFR for PAMB is smaller ( 0.8-0.95) suggesting the anharmonically coupled modes become somewhat decoupled due to the separation of the ring and the azido- group. This lower TFR further explains the observed much weaker IR intensity of the coupled ring modes within the PAMB compound.

In particular, the calculations show that the azido- transition at $2193 \mathrm{~cm}^{-1}$ in PAB couples with a combination band of two modes localized on the ring with vibrational frequencies at $1327 \mathrm{~cm}^{-1}$ and $839 \mathrm{~cm}^{-1}$. Even though the resonance distance is larger than $10 \mathrm{~cm}^{-1}$, the coupling strength is sufficiently large to support the presence of a Fermi resonance. As seen in Table S1, the next highest TFR is $<0.3$ indicating that no other combination bands contribute to the energy pathway.

In the case of the PAMB compound, the computations determine two possible combination bands that likely couple with the azido- transition at $2164 \mathrm{~cm}^{-1}$. The first combination band in resonance with this transition is comprised of a methylene+ring-+azido $\left(1316 \mathrm{~cm}^{-1}\right)$ mode and a ring+methylene $\left(835 \mathrm{~cm}^{-1}\right)$ mode. The second combination band is comprised of a methylene $\left(1217 \mathrm{~cm}^{-1}\right)$ mode and ring+methylene $\left(956 \mathrm{~cm}^{-1}\right)$ mode. The former has the highest third-order coupling constant $\left(\sim 14 \mathrm{~cm}^{-1}\right)$, while the second possibility has the highest TFR ${ }_{\alpha \beta \gamma}$ of $\sim 0.94$, suggesting a possible weak Fermi resonance. These findings are supported by the presence of anharmonic coupling cross peak found in the 2D IR measurements mentioned below. Regardless, the lower magnitudes of the TFR for both cases illustrate that the azido- mode is significantly decoupled from the ring compared to the PAB compound. No other combination bands have a TFR $>0.3$ (as shown in Table S1) indicating no other major contribution to the energy pathway. It should be noted that although there is confidence in the identification of the nature of the coupled modes, the actual resonances could be due to interactions with different ring modes of similar frequency than those identified. 


\section{D IR measurement}

The 2D IR spectra of PAMB and PAB at waiting time $\mathrm{T}=3 \mathrm{ps}$, are shown in Figure $1 \mathrm{C}$ and $1 \mathrm{D}$, respectively. Akin to the linear IR spectrum, the azido- and cyano- $v=0 \rightarrow v=1$ transitions appear along the diagonal at $\omega_{\mathrm{t}}=\omega_{\tau}=2106 \mathrm{~cm}^{-1}$ (peak 1') and $\omega_{\mathrm{t}}=\omega_{\tau}=2228 \mathrm{~cm}^{-1}$ (peak 3') for PAB, respectively. Similar transitions for the PAMB compound are observed along the diagonal at $\omega_{\mathrm{t}}=\omega_{\tau}=2103 \mathrm{~cm}^{-1}$ (peak 1) and $\omega_{\mathrm{t}}=\omega_{\tau}=2230 \mathrm{~cm}^{-1}$ (peak 3). A third transition is observed in the 2D IR spectrum of PAB along the diagonal (peak 9'). This transition is due to a Fermi resonance as indicated by the strong anharmonic coupling cross peaks shown by the rectangular boxes. As mentioned above, it has been shown that the azido- mode strongly couples with a combination of ring mode transitions resulting in a Fermi resonance. Although little evidence of Fermi resonance peaks is present along the diagonal in the 2D IR spectrum of PAMB, a slight elongation of the positive-going azidotransition towards higher energy in $\omega_{\mathrm{t}}$, hilighted by the black rectangle in Figure $1 \mathrm{C}$, is observed. These weak cross peaks suggest some coupling is present between the azidomode and a transition not detectable in the 2D IR spectrum. The absence of a diagonal peak further demonstrates that the fundamental azido- mode is much less coupled to the combination of ring modes.

The negative contours red shifted along the $\omega_{\mathrm{t}}$ axis represent $v=1 \rightarrow v=2$ transitions that occur because of the anharmonic nature of the oscillations. The anharmonicities of the cyano- transition are comparable for both PAMB and PAB at $27 \pm 2 \mathrm{~cm}^{-1}$ (peak 4) and $30 \pm 3$ $\mathrm{cm}^{-1}$ (peak 4'), respectively. The azido- transition for both PAMB and PAB have anharmonicities of approximately $20 \mathrm{~cm}^{-1}$ as shown by peaks 2 and 2' above. The waiting time dependence shows that the peak position of the negative band blue shifts. At $\mathrm{T}=0$ the negative-going transition in PAMB is located at $\omega_{\mathrm{t}}=2068 \mathrm{~cm}^{-1}$ and decays almost completely at $T \approx 2$ ps. At $T=200 \mathrm{fs}$ a second negative band appears at $\omega_{\mathrm{t}}=2084 \mathrm{~cm}^{-1}$ and persists. The azido- transition for PAB shows similar behavior for the negative transition, located at $\omega_{\mathrm{t}}=2077 \mathrm{~cm}^{-1}$ during early times. This transition disappears much faster, revealing a second transition at $\omega_{\mathrm{t}}=2088 \mathrm{~cm}^{-1}$. The presence of dual peaks in the $v=1 \rightarrow v$ $=2$ transition has been reported by Gai and co-workers and described as a corresponding combination band (see SI). ${ }^{79}$ As shown in Figure 1C and 1D, that there is an elongation with the negative contour of the cyano- peak compared to the positive band suggesting that the cyano- mode could be experiencing a similar effect.

In addition to the decaying cross peaks due to the Fermi resonance (black rectangles), several other cross peaks grow in as a function of waiting time. These cross peaks, labelled as 5-8 for PAMB and 5' $-14^{\prime}$ for $\mathrm{PAB}$, result from intramolecular vibrational energy redistribution from one fundamental mode to at least two anharmonically-coupled combination bands of the ring until reaching the final fundamental mode. In PAMB, the energy transfer from the azido- transition to cyano- transition are shown by the cross peaks 5 and 6 . The cross peaks 7 and 8 indicate the possibility of back transfer from cyano- to azido-. Similarly, cross peaks 5' -8 ' represent the same processes in the PAB compound. The aharmonicities between peaks 5 and 6 and peaks $5^{\prime}$ and $6^{\prime}$ is $\sim 12 \mathrm{~cm}^{-1}$. In PAB, cross peaks $11^{\prime}-14^{\prime}$ also arise at the intersection of the Fermi and cyano- transitions. These resonances 
further indicate the modes involved in the vibrational energy transfer pathway. The cross peaks will be analysed and discussed in detail below.

\section{$\mathrm{CN}$ and $\mathrm{N}_{3}$ Vibrational Lifetimes}

The vibrational lifetimes were determined by measuring the peak intensity of the $v=0 \rightarrow v$ $=1$ transitions as a function of the waiting time $\mathrm{T}$. The vibrational lifetime of cyanotransition for $\mathrm{PAB}$ and $\mathrm{PAMB}$ were fit using a single exponential decay, having a decay of $3.69 \pm 0.15$ ps (Fig. 3A) and $3.87 \pm 0.08$ ps (Fig. 3B), respectively.

A bi-exponential fit was utilized to determine the azido- transition lifetime decays of $\mathrm{T}_{10}=0.69 \pm 0.02 \mathrm{ps}$ and $\mathrm{T}^{\prime}{ }_{10}=7.04 \pm 0.07 \mathrm{ps}$ with amplitudes of $\mathrm{A}_{10}=0.85 \pm 0.01$ and $\mathrm{A}^{\prime}{ }_{10}=0.15 \pm 0.01$ for PAMB (Fig. 3B). PAB (Fig. 3A) has similar lifetimes of $\mathrm{T}_{10}=0.83 \pm$ 0.12 ps and $\mathrm{T}^{\prime}{ }_{10}=7.33 \pm 1.40 \mathrm{ps}$ with amplitudes of $\mathrm{A}_{10}=0.71 \pm 0.06$ and $\mathrm{A}^{\prime}{ }_{10}=0.29 \pm 0.05$. The bi-exponential decay is often detected in transitions around this spectral region due to vibrational coupling between the bright mode and spatially close dark modes within the molecule. The fast component is from an equilibrium being reached for the coupling of the bright mode and a dark mode. The longer lifetime is from the vibrational population decay into the ground state. ${ }^{47,} 60,80$ The intensity decay of the Fermi transition along the diagonal also follows a similar trend to that of the azido- transition.

Overall, these experimental vibrational lifetimes are in reasonable agreement with the values calculated with Eq. (1). For PAB, the theoretical values are $4.46 \mathrm{ps}$ and $0.34 \mathrm{ps}$ for the cyano- and azido- transitions, respectively. For PAMB, calculated cyano- transition was determined to be $4.13 \mathrm{ps}$ and the azido- vibrational lifetime was $1.15 \mathrm{ps}$. It should be noted that the experimental vibrational lifetime has two components. However, the fit of the data indicates that the most dominant contribution is from the short component ( $85 \%$ for PAMB and $\sim 70 \%$ for PAB). It is the short component that is captured by Eq. (1), which provides an estimate to the rate constant for the single-exponential decay.

\section{$\mathrm{N}_{3}$ to $\mathrm{CN}$ Cross peaks}

Several cross peaks in the 2D IR spectrum play an important role to expose the energy transfer pathway and the transfer rate. The cross peaks between azido- mode to the cyanomode, observed at $\left\{\omega_{\mathrm{t}}, \omega_{\tau}\right\}=\{2230,2103\} \mathrm{cm}^{-1}$ in PAMB and $\left\{\omega_{\mathrm{t}}, \omega_{\tau}\right\}=\{2228,2106\} \mathrm{cm}$

${ }^{-1}$ for PAB, labelled as 5 and 5 ' in Figure 1, respectively, capture the essence of the processes taking place in the model compounds (Figure 1). At the earliest waiting times, a cross peak is observable, especially for PAMB, suggesting dipolar coupling is occurring (See supporting information). Then, these cross peaks exhibit a growth and a decay in intensity due to IVR from the azido- to cyano- transitions. Although somewhat counterintuitive, the uphill energy flow is possible if the energy separation between vibrational modes is less than $k_{B} T$, where $k_{B}$ is the Boltzmann constant and $T$ is the temperature. ${ }^{58}$ Assuming room temperature $(\sim 293 \mathrm{~K})$, the value is $\sim 200 \mathrm{~cm}^{-1}$ and the energy separation between the two oscillators is $\sim 130 \mathrm{~cm}^{-1}$, suggesting uphill IVR energy flow is allowed. On the other side of the diagonal, the cross peaks, labelled 7 and 8 and 7 ' and 8', appear at T>0, but exhibit an initial lag phaser suggesting some IVR from the cyanoto the azido- mode (Figure 1). The overall decay of these cross peaks represents a 
combination of the initial transfer followed by the thermal cooling of the azido- mode. ${ }^{81}$

Since the cross peaks exhibit only an initial lag phase representing the initial growth before decaying, the vibrational energy flow within these molecules is somewhat masked for the backward transfer. Since the cross peak amplitudes are highly dependent on the characteristics of the two interacting modes, any growth in the cross peaks is likely masked by the significant loss of population in the probed state, the weak dipole moment of the cyano- group, and lack of strong anharmonic coupling with the ring. For more details on the dynamics of cross peaks of back transfer, see the supporting information.

At $\mathrm{T}=0$ no positive cross peak is present in the spectrum of $\mathrm{PAB}$, but instead an elongated negative peak is observed from $\omega_{\mathrm{t}} \approx 2190 \mathrm{~cm}^{-1}$ to $\omega_{\mathrm{t}} \approx 2240 \mathrm{~cm}^{-1}$ as shown in Figure $\mathrm{S} 2$. The positive transition finally appears after $T \approx 1.6 \mathrm{ps}$. There are several possible reasons for the origin of the elongated negative-going transition. One possibility is that the transition originates from fast vibrational energy transfer from the azido- mode to dark transitions found within the ring. Oftentimes in the IVR process, a negative cross peak arises due to dark modes coupling with the transition that receives the energy (cyano- mode). ${ }^{19}$ Due to the strong Fermi resonance, the energy flow from the azido- to the dark transitions is much faster compared to that of the dark transitions to the cyano- mode. As a result, a broad negative peak appears indicating a delayed energy relaxation into the cyano- mode. Over time, the positive transition appears as the energy finally relaxes to final mode.

PAMB has a weaker Fermi resonance slowing the energy transition from the azido- mode to the dark states. The coupling strength between the cyano- and combination bands are similar for both PAB and PAMB where the anharmonicity of the cross peaks are $12 \mathrm{~cm}^{-1}$. Thus, the IVR transfer from the azido- to the cyano- mode in PAMB is slower, allowing the positivegoing cross peak not to be masked as discussed above for PAB. An oscillatory feature is observed in the cross peak decay. It is likely due to vibrational beating between transitions with an oscillating frequency of $\sim 13 \mathrm{~cm}^{-1}$.

\section{Cross Peaks Dynamics}

At the intersection of the azido- and cyano- transitions, cross peaks appear in the off diagonal at later waiting times, $T>0$ as a result of IVR between the transitions. Peaks 5 and 5 ' in Figure 1 show both a growth and a decay. Since dipolar coupling is detected in PAMB within the same spectral region as the IVR, it can influence the growth and decay rate of the cross peaks during the IVR process. However, with such a short lifetime ( $\sim 300 \mathrm{fs})$, the contributions of dipolar coupling are essentially gone ( $5 \%$ at $800 \mathrm{fs})$ before the intensity of the cross peaks starts to increase (see SI). Thus, in this case, the influence is not significant.

Intramolecular vibrational energy redistribution between vibrational modes occurs in polyatomic molecules by energy transport from one mode to the others via multiple anharmonically coupled low frequency modes. Knowing the pathway for energy transfer is difficult as the coupling is not just between the two modes of interest but instead it involves several combination bands and other dark modes that are not easily detected experimentally. However, analyzing the rate of energy transfer and comparing these results to theory can aid in the identification of such modes and create new insights into the mechanism involved. It should be noted that cross peaks occasionally arise through thermal transfer which result in 
frequency shifts indistinguishable from direct excitation of the accepting mode. ${ }^{19}$ Still, this thermal response is typically observed in larger systems, and thus it is unlikely for these small model systems. However, we cannot completely rule out some contribution from this effect.

One approach to quantify the rate of energy transfer is to measure where the cross peak intensity reaches a maximum, as demonstrated by the Rubtsov group. ${ }^{19,}$, 48, 53, 62-63, 65, 81 .

Figure 4 shows the growth and decay of the positive cross peak intensities for both PAB and PAMB. In this model the time it takes for the cross peak intensity to reach a maximum is the vibrational population transport time from the azido- to the cyano-. The decay time represents cooling of the cyano- mode. ${ }^{81}$ For PAMB, the cross peak intensity begins to increase at $\mathrm{T}=800 \mathrm{fs}$ and exhibits a relatively smooth growth and decay. For PAB, the cross peak appears much later at $\mathrm{T}=1.6 \mathrm{ps}$. As mentioned earlier, the negative cross peak is broad for PAB at early waiting times and overlaps with the positive peak interfering with the intensity. Thus, the positive cross peak growth and decay exhibits more noise. Initially, both intensity profiles were fitted with an unconstrained bi-exponential growth and decay function to measure the time of maximal intensity. The original growth rate for PAMB is half of PAB suggesting multiple pathways for IVR. ${ }^{62}$ The decay rates were similar which is expected due to the similar vibrational lifetimes of the cyano- transition. The data were then fitted with a constrained bi-exponential, which is shown in Figure 4, where the decay rate was constrained to the vibrational lifetime of the cyano-. The growth rate of PAMB was still lower than that of PAB.

As shown in the graph, PAMB reaches a maximum prior to $\mathrm{PAB}$ which does not initially make sense as the pathway from the azido- to the cyano- is shorter. However, while a correlation between bond distance and time of maximum spectral amplitude have been covered in previous experiments, ${ }^{48,53,63}$ the structure of the molecule can also influence the rate as well, along with bond distance which has been shown in other benzene systems. ${ }^{62}$ The structural flexibility influences the overlap between the modes of the system thus influencing the rate of energy transfer. In our case the methylene group creates a difference between the two molecules where the azido- in PAMB can exhibit more movement (such as a rotation or wiggle). Also, the negative band present at early waiting times for PAB does influence the amplitude of the growth function. In the bi-exponential fit for PAMB the amplitudes are essentially equal where at $\mathrm{T}=0$ the cross peak intensity is approximately zero which is expected. When the amplitude of the growth function is set equal to the amplitude of the decay constant the $T_{\max }$ was recalculated for PAB yielding a value of $T_{m a z}=3.1$ ps. The corresponding transport rate is $227 \mathrm{~m} / \mathrm{s}$. For PAMB the $\mathrm{T}_{\max }$ was determined to be 4.6 ps, resulting in a transport speed of $186 \mathrm{~m} / \mathrm{s}$ (see SI for details). ${ }^{19}$

\section{Kinetics}

Although using the cross peak intensity maximum has been shown as an effective method to measure rates of energy transfer, another approach presented in the literature is a simple kinetic model which relates the vibrational lifetimes with the rates of energy transfer from one transition to another. ${ }^{21}$ In our case this model can be represented as the following scheme: 


$$
\stackrel{k_{N}}{\longleftarrow} N_{3} \stackrel{K_{e q}}{\longleftrightarrow} C N \stackrel{k_{C N}}{\longrightarrow}
$$

where $k_{N_{3}}$ and $k_{C N}$ are the experimentally measured vibrational decay constants of azidoand cyano-, respectively. $k_{C N} \rightarrow N_{3}$ is the energy transfer rate (as indicated by the cross peaks 7 and 7 ' in Figure 1) from the cyano- to azido- mode and $k_{N_{3} \rightarrow C N}$ is the energy transfer rate from the azido- to the cyano- mode. In both cases, the azido- lifetime was biexponential in nature with a slow component and a fast one. The fast component is not used in the analysis because of the time scale of the cross peak dynamics. The cross peaks arise around $\mathrm{T} \approx 1 \mathrm{ps}$ and the fast lifetime component is much faster, $\mathrm{T}<1 \mathrm{ps}$. The fast component simply allows the fundamental and dark modes to reach an equilibrium and reduces the population of the azido- mode (down to $29 \%$ for PAB and $15 \%$ for PAMB), thus the population in the kinetic model was adjusted appropriately. ${ }^{80}$ The long component is the rate determining step in the IVR process and used in the kinetic scheme.

Since the vibrational lifetimes are directly measured experimentally, the energy transfer rates, $k_{N_{3} \rightarrow C N}$ and $k_{C N} \rightarrow N_{3}$, are determined from the fit of the kinetic model. The rate constants are related by detailed balance:

$$
K_{e q}=\frac{k_{N_{3} \rightarrow C N}}{k_{C N} \rightarrow N_{3}}=\exp \left(-\frac{E_{C N}-E_{N_{3}}}{R T}\right)
$$

where $\mathrm{R}$ is the Boltzmann constant, $E_{C N}$ and $E_{N_{3}}$ are the respective excitation energies, and the temperature is $293 \mathrm{~K}$ (approximately the room temperature). With $k_{N_{3} \rightarrow C N}$ and $k_{C N} \rightarrow N_{3}$ as the fitting variables, the principle of detail balance allows for substitution of $k_{N_{3} \rightarrow C N}=k_{e q}{ }^{*} k_{C N} \rightarrow N_{3}$. The details of the model are elaborated in the SI.

A least-squares fit was performed for the growth and decay of the cross peak intensity (CP) of 5 and 5' in Figure 1 and is shown in Figure 5. The following equations were utilized to obtain the IVR rate from the azido- to the cyano- mode

$$
\begin{gathered}
C P=\frac{X Y N_{3_{0}}}{X-Y}\left[-e^{\lambda_{1} t}+3.5 * e^{\lambda_{2} t}\right] \\
C P=\frac{X Y N_{3_{0}}}{X-Y}\left[-e^{\lambda_{1} t}+e^{\lambda_{2} t}\right]
\end{gathered}
$$

Equation 3 was used for the intensity of cross peak 5' of the PAB compound, while equation 4 was used for the fit of the intensity cross peak 5 for the PAMB compound. The amplitudes for the growth and the decay of the kinetic trace should be equal since the energy transfer into the mode should be the same as the energy lost as the mode cools. However, for PAB they are not. Thus, a weighting factor of 3.5 in equation 3 was added to account for the 
difference in amplitudes in the growth and decay exponential rates discussed above (see SI for details). $N_{3_{0}}$ is the population of the azido- mode after the initial equilibrium. To account for any loss of population due to the initial fast equilibrium during the vibrational relaxation, a factor (29\% for PAB and $15 \%$ for PAMB) was multiplied by the amplitude from the slow component in the bi-exponential decay. In equation 3 and 4

$$
\lambda_{1}=\frac{\left(K_{1}+K_{2}\right)+\sqrt{\left(K_{1}-K_{2}\right)^{2}+4\left(k_{N_{3}} \rightarrow C N\right)\left(k_{C N} \rightarrow N_{3}\right)}}{2},
$$

$$
\lambda_{2}=\frac{\left(K_{1}+K_{2}\right)-\sqrt{\left(K_{1}-K_{2}\right)^{2}+4\left(k_{N_{3}} \rightarrow C N\right)\left(k_{C N} \rightarrow N_{3}\right)}}{2},
$$

$$
X=\frac{\left(-K_{1}+K_{2}\right)+\sqrt{\left(K_{1}-K_{2}\right)^{2}+4\left(k_{N_{3}} \rightarrow C N\right)\left(k_{C N} \rightarrow N_{3}\right)}}{2 k_{C N} \rightarrow N_{3}},
$$

$Y=\frac{\left(-K_{1}+K_{2}\right)-\sqrt{\left(K_{1}-K_{2}\right)^{2}+4\left(k_{N_{3}} \rightarrow C N\right)\left(k_{C N} \rightarrow N_{3}\right)}}{2 k_{C N} \rightarrow N_{3}}$, and $K_{1}=-k_{N_{3}}-k_{N_{3} \rightarrow C N}$ and $K_{2}=-k_{C N}-k_{C N} \rightarrow N_{3}$. A fit to the experimental data was achieved by variation of only the $k_{C N} \rightarrow N_{3}$ parameter. From the least squares fit, the azido- to cyano- transfer rate is $k_{N_{3} \rightarrow C N}=0.540 \pm 0.151 \mathrm{ps}^{-1}\left(\tau_{N_{3} \rightarrow C N}=1.9 \pm 0.532 \mathrm{ps}\right)$ for PAB, and $k_{N_{3} \rightarrow C N}=0.0434 \pm 0.0109 \mathrm{ps}^{-1}\left(\tau_{N_{3} \rightarrow C N}=23.0 \pm 5.75 \mathrm{ps}\right)$ for PAMB. As expected, the transfer rate is faster for PAB compared to PAMB (See SI for back transfer data and rates).

To compare these results to our theoretical studies, the IVR rate was determined by adding the calculated IVR rates for each of the components along the proposed pathway; i.e. the IVR rate from the azido- to the Fermi resonance and the combination bands within the ring to cyano- (via microscopic reversibility) with the strongest coupling. While multiple possible pathways likely exist for IVR, only the most probable ones are considered with the highest TFR value. In PAB, the IVR rate from the azido- to the combination band is $k_{N_{3}} \rightarrow$ Fermi $=2.6 \mathrm{ps}^{-1}\left(\tau_{N_{3}} \rightarrow\right.$ Fermi $\left.=0.38 \mathrm{ps}\right)$ and the IVR rate from the combination band to the cyano- is $k_{R i n g} \rightarrow C N=0.068 \mathrm{ps}^{-1}$ ( $\tau_{R i n g} \rightarrow C N=14.8 \mathrm{ps}$ ) which is much slower due to being delocalized. Thus, the total energy transfer rate is determined by the following equation:

$$
\frac{1}{k_{N_{3} \rightarrow C N}}=\frac{1}{k_{N_{3} \rightarrow \text { Fermi }}}+\frac{1}{k_{\text {Ring } \rightarrow C N}}
$$

yielding a transfer rate of $k_{N_{3} \rightarrow C N}=0.066 \mathrm{ps}^{-1}\left(\tau_{N_{3} \rightarrow C N}=15.1 \mathrm{ps}\right)$.

As for the PAMB compound, the theoretical energy transfer rate from the azido- to the cyano- mode was calculated in a similar fashion. However, as discussed earlier, there are two 
highly probable pathways from the azido- to the cyano- based on the values of the $T F R_{a \beta \gamma}$., $\Phi_{\mathrm{a} \beta \gamma}$, and $\Delta \omega$. The figure of merit for strongly anharmonically coupled modes is the $T F R_{a \beta \gamma}$ as it considers all the relevant parameters. The IVR rate from the ring to the cyanomode is similar to the PAB pathway, having a value of $k_{R i n g} \rightarrow C N=0.09 \mathrm{ps}^{-1}$

( $\tau_{\text {Ring } \rightarrow C N}=11.1 \mathrm{ps}$ ). The two possibilities for the energy to flow from the azido- to the ring have IVR rates of $k_{N_{3}} \rightarrow$ Fermi $=0.26 \mathrm{ps}^{-1}\left(\tau_{N_{3}} \rightarrow\right.$ Fermi $\left.=3.9 \mathrm{ps}\right)$ and $k^{\prime} N_{3} \rightarrow$ Fermi $=0.05 \mathrm{ps}^{-1}$

$\left(\tau^{\prime} N_{3} \rightarrow\right.$ Fermi $\left.=18.3 \mathrm{ps}\right)$. Since both of these combination bands have a $T F R_{a \beta \gamma}>0.7$ (See

Table 1), each mode was analysed as a part of the primary pathway of energy transfer giving a range of IVR times from 15 to 33 ps. The experimental value falls well within this range suggesting that the major contributions to the pathway have been identified. To further refine the model, an approach to include both competing pathways was instituted to acquire a theoretical rate. In this approach, the contribution of each competing pathway was weighted by the calculated TFR values, since these values represent the strength of the anharmonic coupling between the azido- and the combination bands. Since the combined total TFR value for two pathways is 1.716 (a similar value to $\mathrm{PAB}$ ), the fractional contribution of each pathway would be 0.45 and 0.55 for the $T F R_{a \beta \gamma}$ values of 0.77 and 0.94 , respectively. With these weighted contributions, the IVR time for the combined pathway would be $\tau_{N_{3} \rightarrow \text { Fermi }}$ $=0.45 * 3.9 \mathrm{ps}+0.55 * 18.3 \mathrm{ps}=11.8 \mathrm{ps}$. With this value, the total transport time, $\tau=\tau_{N_{3} \rightarrow \text { Fermi }}+\tau^{\prime} N_{3} \rightarrow$ Fermi $+\tau_{\text {Ring } \rightarrow C N}$, is 22.9 ps. Through this combined transfer pathway model, the IVR process matches well with experiment and thus the pathway is identified.

The overall trend in the theoretical rate constants matches the experimental results, i.e. PAB is faster. Furthermore, the magnitude of the experimental and theoretical constants for the PAMB is within $<1 \%$ showing that our simplistic approach captures the most important contributions of the energy transfer pathway. Due to significant congestion in the cross peak region of the experiment, the theoretical and experimental rates show a much larger deviation ( 7-fold) for the PAB compound. In particular, the major overlap occurs when the negative-going cross peak, present at $\mathrm{T}=0$, cancels out the positive-going cross peak until much later waiting times. As a result of this spectral overlap, the initial slope of the PAB kinetic trace rises more rapidly than PAMB. Thus, it creates an artificial measure of the rate constant and deviates from the actual IVR growth rate. Despite the difference from the theoretical values, a singular pathway can be identified due to the large $T F R_{a \beta \gamma}(>1.9)$ for only one combination band. In PAMB, with less congestion is observed in the cross peak region of the 2D IR spectrum. Thus, the positive-going cross peak is always clearly present and exhibit a smooth growth and decay of similar amplitudes, unlike the PAB.

Overall, the strength of the Fermi resonance depends on the energetic degeneracy of the fundamental and combination bands that are anharmonically coupled. Stronger coupling between modes results in a pathway for vibrational energy to propagate within the molecule. Both our experimental and theoretical results indicate that a stronger Fermi resonance is present in PAB, leading to a faster IVR rate. Although PAMB also exhibits some anharmonic coupling as shown in both the FTIR and 2D IR, the IVR rate of transfer is significantly slower. Due to the lack of strong coupling in PAMB, the energy transfer 
pathway requires multiple pathways (at least two) from the azido- mode to propagate through the molecule to the cyano- mode.

By reducing the coupling strength via the addition of a single carbon atom between the ring and the azido- group, the resulting bottleneck effect not only decreases the rate of IVR but still maintains slightly favorable energy flow in the forward direction. It should be noted that tuning the Fermi resonance strength, the propensity of energy to flow in one direction can become either less or more favorable. Developing methods to modify energy transfer in this way will allow reactivity to be guided for desired chemical reactions and product formation. During chemical reactions, energy is transported via vibrations from one nucleus to another. Modifying this energy flow can lead to more efficient chemical synthesis. This work and previous literature show that the rate of energy transfer can be tuned by the strength of the Fermi resonance. ${ }^{44-45}$

Also, the IVR studied here is relevant in the design of energy diodes. ${ }^{30,}{ }^{39}$ In particular, our studies demonstrate that vibrational energy rectification can be exploited for molecular electronics.

\section{Conclusions}

Intermolecular vibrational energy redistribution has the power to influence the underlying fundamental principles of chemistry ranging from chemical reactivity to energy flow in diodes. In this manuscript, we have described the details of the coupling processes for these systems and demonstrated that molecular energy flow is tunable via alteration of Fermi resonances and the intrinsic coupling manifold of the molecule. Serving as a model system, this approach for uncovering the coupling manifold between these two transitions expands the notion that the transfer pathway can be influenced to guide the energy flow and rectification, which is necessary for design of molecular electronics. In addition, with a better understanding of the mechanisms at work, the IVR pathway can be limited to one favorable direction making molecular selectivity in synthesis and biological function become possible.

In this work, the energy transfer pathways and rates were determined for both PAB and PAMB compounds. With the similarity in scaffold, the rate was modulated by variation of the coupling present along the transfer pathway. Thus, the transfer time in PAB $(\sim 2.0 \mathrm{ps})$ was slowed by more than an order of magnitude via only a slight change in the coupling, i.e. localization of the azido group. For PAMB (IVR $23.0 \mathrm{ps}$ ), the methylene group decoupled the azido- mode from the ring to modulate the strength of the Fermi resonance that guides the energy transfer pathway. These results are substantiated by the theoretical calculations indicating a decrease in the TFR and by the experimental results showing a decrease of Fermi coupling peaks in the 2D IR spectrum. Our results provide a systematic roadmap to vary energy transfer within an aromatic scaffold by modulating the associated Fermi resonances, showing that the simple addition of a methylene group can slow down energy transfer rate by $>10$ fold. 
In addition to varying the rate of transfer, the direction of the IVR pathway from the azidoto cyano- can be somewhat regulated via the presence of a strong Fermi resonance. The Fermi resonance with the azido- group formally locks in the fast transfer on one end of the molecular scaffold, while the lack of the Fermi resonance slightly inhibits back flow on the other side. Even in the PAMB compound where two pathways are necessary, the presence of the weaker Fermi resonance is enough to cause the pathway to remain slightly favored. As for comparison to the literature, the correlation between the transport time and distance uncovered by Rubtsov ${ }^{62}$ was well within the typical range found for IVR in other scaffolds (150 to $500 \mathrm{~m} / \mathrm{s}$ ). Modifying the Fermi resonance alters the energy flow from one transition to another. These subtle changes can translate to changes in chemical synthesis. Varying the energy dissipation within the molecular system can improve/reduce the required energy of a reactive pathway leading to selectivity. Recently, for example, Scholes and coworkers found that a terpyridine-molybdenum complex containing a dinitrogen bridge exhibits a Fermi resonance that can be activated by light to transfer energy to the spatially separated dinitrogen, facilitating reactions involving $\mathrm{N}_{2} \cdot 45$

Using the approach put forth in this manuscript, the possible transport pathways can be systematically evaluated for their contributions to the overall transfer rate. During the analysis, the experimental rates are compared to the theoretical rates determined from each possible pathway, guided by the third order resonance factor, $T F R_{a \beta \gamma}$. Ultimately, the closest match to the experimental value of the transfer rate reveals the major contributing pathway. In the PAMB model system, the theoretical rate constant matches almost exactly the experimental values, showing the strength of this combined approach. Unfortunately, for the PAB model system, although the pathway is easier to identify from theory, the approach of matching the rate constants is complicated by the congestion in the cross peak region, leading to less accuracy in matching the experimental values. Overall, our work shows that with a combination of experiment and theoretical analysis, the IVR pathway can be determined and the rate subsequently tuned by modulation of the coupling manifold within molecular systems.

\section{Supplementary Material}

Refer to Web version on PubMed Central for supplementary material.

\section{ACKNOWLEDGMENT}

We thank Gwendolyn Fowler for the synthesis of PAMB.

Funding Sources

The research was supported by NIH (R15GM1224597) to MJT, NSF CHE-1361776 and CHE-1854271 to DML, Henry Dreyfus Teacher-Scholar Award (TH-15-009) to SHB, and NIH (R15GM093330) to SHB/EEF.

\section{REFERENCES}

1. Hamm P; Lim M; DeGrado WF; Hochstrasser RM, The two-dimensional IR nonlinear spectroscopy of a cyclic penta-peptide in relation to its three-dimensional structure. Proc. Natl. Acad. Sci. U. S. A 1999, 96 (5), 2036-2041. [PubMed: 10051590] 
2. Hamm P; Lim MH; Hochstrasser RM, Structure of the amide I band of peptides measured by femtosecond nonlinear-infrared spectroscopy. J. Phys. Chem. B 1998, 102 (31), 6123-6138.

3. Mandal A; Pack GN; Shah PP; Erramilli S; Ziegler LD, Ultrafast Two-Dimensional Infrared Spectroscopy of a Quasifree Rotor: J Scrambling and Perfectly Anticorrelated Cross Peaks. Phys. Rev. Lett 2018, 120 (10).

4. Chalyavi F; Gilmartin PH; Schmitz AJ; Fennie MW; Tucker MJ, Synthesis of 5-Cyano-Tryptophan as a 2D Infrared Spectroscopic Reporter of Structure. Angew. Chem. Int. Ed 2018, 57 (25), 75287532.

5. Schmitz AJ; Hogle DG; Gai XS; Fenlon EE; Brewer SH; Tucker MJ, Two-Dimensional Infrared Study of Vibrational Coupling between Azide and Nitrile Reporters in a RNA Nucleoside. J. Phys. Chem. B 2016, 120 (35), 9387-9394. [PubMed: 27510724]

6. Kratochvil HT; Carr JK; Matulef K; Annen AW; Li H; Maj M; Ostmeyer J; Serrano AL; Raghuraman H; Moran SD; Skinner JL; Perozo E; Roux B; Valiyaveetil FI; Zanni MT, Instantaneous ion configurations in the $\mathrm{K}+$ ion channel selectivity filter revealed by $2 \mathrm{D}$ IR spectroscopy. Science 2016, 353 (6303), 1040-1044. [PubMed: 27701114]

7. Anna JM; Kubarych KJ, Watching solvent friction impede ultrafast barrier crossings: A direct test of Kramers theory. J. Chem.Phys 2010, 133 (17).

8. Bagchi S; Charnley AK; Smith AB; Hochstrasser RM, Equilibrium Exchange Processes of the Aqueous Tryptophan Dipeptide. J. Phys. Chem. B 2009, 113 (24), 8412-8417. [PubMed: 19459617]

9. Kim YS; Hochstrasser RM, Chemical exchange 2D IR of hydrogen-bond making and breaking. Proc. Natl. Acad. Sci. U. S. A 2005, 102 (32), 11185-11190. [PubMed: 16040800]

10. Asplund MC; Zanni MT; Hochstrasser RM, Two-dimensional infrared spectroscopy of peptides by phase-controlled femtosecond vibrational photon echoes. Proc. Natl. Acad. Sci. U. S. A 2000, 97 (15), 8219-8224. [PubMed: 10890905]

11. Zheng JR; Kwak K; Asbury J; Chen X; Piletic IR; Fayer MD, Ultrafast dynamics of solute-solvent complexation observed at thermal equilibrium in real time. Science 2005, 309 (5739), 1338-1343. [PubMed: 16081697]

12. Chalyavi F; Hogle DG; Tucker MJ, Tyrosine as a Non-perturbing Site-Specific Vibrational Reporter for Protein Dynamics. J. Phys. Chem. B 2017, 121 (26), 6380-6389. [PubMed: 28590738]

13. DeFlores LP; Ganim Z; Ackley SF; Chung HS; Tokmakoff A, The anharmonic vibrational potential and relaxation pathways of the amide I and II modes of N-methylacetamide. J. Phys. Chem. B 2006, 110 (38), 18973-18980. [PubMed: 16986892]

14. Krummel AT; Zanni MT, DNA vibrational coupling revealed with two-dimensional infrared spectroscopy: Insight into why vibrational spectroscopy is sensitive to DNA structure. J. Phys. Chem. B 2006, 110 (28), 13991-14000. [PubMed: 16836352]

15. Scheurer C; Mukamel S, Infrared analogs of heteronuclear nuclear magnetic resonance coherence transfer experiments in peptides. J. Chem.Phys 2002, 116 (15), 6803-6816.

16. Woys AM; Almeida AM; Wang L; Chiu CC; McGovern M; de Pablo JJ; Skinner JL; Gellman SH; Zanni MT, Parallel beta-Sheet Vibrational Couplings Revealed by 2D IR Spectroscopy of an Isotopically Labeled Macrocycle: Quantitative Benchmark for the Interpretation of Amyloid and Protein Infrared Spectra. J. Am. Chem. Soc 2012, 134 (46), 19118-19128. [PubMed: 23113791]

17. Remorino A; Hochstrasser RM, Three-Dimensional Structures by Two-Dimensional Vibrational Spectroscopy. Acc. Chem. Res 2012, 45 (11), 1896-1905. [PubMed: 22458539]

18. Hamm P; Zanni MT, Concepts and Methods of 2D Infrared Spectroscopy Cambridge University Press: 2011.

19. Rubtsova NI; Rubtsov IV, Vibrational Energy Transport in Molecules Studied by RelaxationAssisted Two-Dimensional Infrared Spectroscopy. In Annu. Rev. Phys. Chem, Johnson MA; Martinez TJ, Eds. 2015; Vol. 66, pp 717-738. [PubMed: 25747112]

20. Cahoon JF; Sawyer KR; Schlegel JP; Harris CB, Determining transition-state geometries in liquids using 2D-IR. Science 2008, 319 (5871), 1820-1823. [PubMed: 18369145] 
21. Anna JM; King JT; Kubarych KJ, Multiple Structures and Dynamics of $[\mathrm{CpRu}(\mathrm{CO})(2)](2)$ and $[\mathrm{CpFe}(\mathrm{CO})(2)](2)$ in Solution Revealed with Two-Dimensional Infrared Spectroscopy. Inorg. Chem 2011, 50 (19), 9273-9283. [PubMed: 21875040]

22. Bian HT; Wen XW; Li JB; Zheng JR, Mode-specific intermolecular vibrational energy transfer. II. Deuterated water and potassium selenocyanate mixture. J. Chem.Phys 2010, 133 (3).

23. He XM; Yu PY; Zhao J; Wang JP, Efficient Vibrational Energy Transfer through Covalent Bond in Indigo Carmine Revealed by Nonlinear IR Spectroscopy. J. Phys. Chem. B 2017, 121 (40), 94119421. [PubMed: 28922919]

24. Kurochkin DV; Naraharisetty SRG; Rubtsov IV, A relaxation-assisted 2D IR spectroscopy method. Proc. Natl. Acad. Sci. U. S. A 2007, 104 (36), 14209-14214. [PubMed: 17557837]

25. Leitner DM, Energy flow in proteins. In Annu. Rev. Phys. Chem, 2008; Vol. 59, pp 233-259. [PubMed: 18393676]

26. Leitner DM, Mode damping rates in a protein chromophore. Chem. Phys. Lett 2012, 530, 102-106.

27. Pandey HD; Leitner DM, Vibrational energy transport in molecules and the statistical properties of vibrational modes. Chem. Phys 2017, 482, 81-85.

28. Pandey HD; Leitner DM, Influence of thermalization on thermal conduction through molecular junctions: Computational study of PEG oligomers. J. Chem.Phys 2017, 147 (8).

29. Leitner DM; Pandey HD, Asymmetric energy flow in liquid alkylbenzenes: A computational study. J. Chem.Phys 2015, 143 (14).

30. Leitner DM; Pandey HD, Quantum bottlenecks and unidirectional energy flow in molecules. Annalen Der Physik 2015, 527 (9-10), 601-609.

31. Yu X; Leitner DM, Vibrational energy transfer and heat conduction in a protein. J. Phys. Chem. B 2003, 107 (7), 1698-1707.

32. Botan V; Backus EHG; Pfister R; Moretto A; Crisma M; Toniolo C; Nguyen PH; Stock G; Hamm P, Energy transport in peptide helices. Proc. Natl. Acad. Sci. U. S. A 2007, 104 (31), 1274912754. [PubMed: 17646650]

33. Fujisaki H; Stock G, Dynamic treatment of vibrational energy relaxation in a heterogeneous and fluctuating environment. J. Chem.Phys 2008, 129 (13).

34. Nguyen PH; Park SM; Stock G, Nonequilibrium molecular dynamics simulation of the energy transport through a peptide helix. J. Chem.Phys 2010, 132 (2).

35. Rubtsov IV; Hochstrasser RM, Vibrational dynamics, mode coupling, and structural constraints for acetylproline-NH2. J. Phys. Chem. B 2002, 106 (35), 9165-9171.

36. Owrutsky JC; Raftery D; Hochstrasser RM, Vibrational-Relaxation Dynamics in Solutions Annu. Rev. Phys. Chem 1994, 45, 519-555. [PubMed: 7811356]

37. Elsaesser T; Kaiser W, Vibrational and Vibronic Relaxation of Large Polyatomic-Molecules in Liquids. Annu. Rev. Phys. Chem 1991, 42, 83-107.

38. Pein BC; Dlott DD, Modifying Vibrational Energy Flow in Aromatic Molecules: Effects of Ortho Substitution. J. Phys. Chem. A 2014, 118 (6), 965-973. [PubMed: 24428786]

39. Pein BC; Sun YX; Dlott DD, Unidirectional Vibrational Energy Flow in Nitrobenzene. J. Phys. Chem. A 2013, 117 (29), 6066-6072. [PubMed: 23432106]

40. Bian HT; Zhao W; Zheng JR, Intermolecular vibrational energy exchange directly probed with ultrafast two dimensional infrared spectroscopy. J. Chem.Phys 2009, 131 (12).

41. Dong XQ; Yang F; Zhao J; Wang JP, Efficient Intramolecular Vibrational Excitonic Energy Transfer in Ru-3(CO)(12) Cluster Revealed by Two-Dimensional Infrared Spectroscopy. J. Phys. Chem. B 2018, 122 (3), 1296-1305. [PubMed: 29275631]

42. Yang F; Zhao J; Wang JP, Two-Dimensional Infrared Study of C-13-Natural Abundant Vibrational Transition Reveals Intramolecular Vibrational Redistribution Rather than Fluxional Exchange in Mn(CO)(5)Br. J. Phys. Chem. B 2016, 120 (7), 1304-1311. [PubMed: 26836759]

43. Bian HT; Li JB; Wen XW; Zheng JR, Mode-specific intermolecular vibrational energy transfer. I. Phenyl selenocyanate and deuterated chloroform mixture. J. Chem.Phys. 2010, 132 (18).

44. Leitner DM, Illuminating Fermi Resonances that Trigger Reaction in a Complex Molecule. Chem 2019, 5 (2), 256-257. 
45. Rafiq S; Bezdek MJ; Chirik PJ; Scholes GD, Dinitrogen Coupling to a Terpyridine-Molybdenum Chromophore Is Switched on by Fermi Resonance. Chem 2019, 5 (2), 402-416.

46. Leitner DM, Quantum ergodicity and energy flow in molecules. Adv. Phys 2015, 64 (4), 445-517.

47. Bian HT; Wen XW; Li JB; Chen HL; Han SZ; Sun XQ; Song JA; Zhuang W; Zheng JR, Ion clustering in aqueous solutions probed with vibrational energy transfer. Proc. Natl. Acad. Sci. U. S. A 2011, 108 (12), 4737-4742.

48. Kasyanenko VM; Lin ZW; Rubtsov GI; Donahue JP; Rubtsov IV, Energy transport via coordination bonds. J. Chem.Phys 2009, 131 (15).

49. Lin ZW; Zhang N; Jayawickramarajah J; Rubtsov IV, Ballistic energy transport along PEG chains: distance dependence of the transport efficiency. Phys. Chem. Chem. Phys 2012, 14 (30), 1044510454. [PubMed: 22555778]

50. Chen HL; Bian HT; Li JB; Wen XW; Zhang Q; Zhuang W; Zheng JR, Vibrational Energy Transfer: An Angstrom Molecular Ruler in Studies of Ion Pairing and Clustering in Aqueous Solutions. J. Phys. Chem. B 2015, 119 (12), 4333-4349. [PubMed: 25679402]

51. Bian HT; Chen HL; Li JB; Wen XW; Zheng JR, Nonresonant and Resonant Mode-Specific Intermolecular Vibrational Energy Transfers in Electrolyte Aqueous Solutions. J. Phys. Chem. A 2011, 115 (42), 11657-11664. [PubMed: 21916443]

52. Li JB; Bian HT; Chen HL; Wen XW; Hoang BT; Zheng JR, Ion Association in Aqueous Solutions Probed through Vibrational Energy Transfers among Cation, Anion, and Water Molecules. J. Phys. Chem. B 2012, 117 (16), 4274-4283. [PubMed: 22928938]

53. Rubtsov IV, Relaxation-Assisted Two-Dimensional Infrared (RA 2DIR) Method: Accessing Distances over 10 angstrom and Measuring Bond Connectivity Patterns. Acc. Chem. Res 2009, 42 (9), 1385-1394. [PubMed: 19462972]

54. Backus EHG; Nguyen PH; Botan V; Moretto A; Crisma M; Toniolo C; Zerbe O; Stock G; Hamm P, Structural Flexibility of a Helical Peptide Regulates Vibrational Energy Transport Properties. J. Phys. Chem. B 2008, 112 (48), 15487-15492. [PubMed: 18991434]

55. Muller-Werkmeister HM; Bredenbeck J, A donor-acceptor pair for the real time study of vibrational energy transfer in proteins. Phys. Chem. Chem. Phys 2014, 16 (7), 3261-3266. [PubMed: 24413252]

56. Muller-Werkmeister HM; Li YL; Lerch EBW; Bigourd D; Bredenbeck J, Ultrafast Hopping from Band to Band: Assigning Infrared Spectra based on Vibrational Energy Transfer. Angew. Chem. Int. Ed 2013, 52 (24), 6214-6217.

57. King JT; Ross MR; Kubarych KJ, Water-Assisted Vibrational Relaxation of a Metal Carbonyl Complex Studied with Ultrafast 2D-IR. J. Phys. Chem. B 2012, 116 (12), 3754-3759. [PubMed: 22376278]

58. King JT; Anna JM; Kubarych KJ, Solvent-hindered intramolecular vibrational redistribution. Phys. Chem. Chem. Phys 2011, 13 (13), 5579-5583. [PubMed: 21359345]

59. Fedoseeva M; Delor M; Parker SC; Sazanovich IV; Towrie M; Parker AW; Weinstein JA, Vibrational energy transfer dynamics in ruthenium polypyridine transition metal complexes. Phys. Chem. Chem. Phys 2015, 17 (3), 1688-1696. [PubMed: 25463745]

60. Kaziannis S; Wright JA; Candelaresi M; Kania R; Greetham GM; Parker AW; Pickett CJ; Hunt NT, The role of $\mathrm{CN}$ and $\mathrm{CO}$ ligands in the vibrational relaxation dynamics of model compounds of the [FeFe]-hydrogenase enzyme. Phys. Chem. Chem. Phys 2011, 13 (21), 10295-10305. [PubMed: 21537507]

61. Delor M; Sazanovich IV; Towrie M; Spall SJ; Keane T; Blake AJ; Wilson C; Meijer AJHM; Weinstein JA, Dynamics of Ground and Excited State Vibrational Relaxation and Energy Transfer in Transition Metal Carbonyls. J. Phys. Chem. B 2014, 118 (40), 11781-11791. [PubMed: 25198700]

62. Kasyanenko VM; Tesar SL; Rubtsov GI; Burin AL; Rubtsov IV, Structure Dependent Energy Transport: Relaxation-Assisted 2DIR Measurements and Theoretical Studies. J. Phys. Chem. B 2011, 115 (38), 11063-11073. [PubMed: 21859144]

63. Naraharisetty SRG; Kasyanenko VM; Rubtsov IV, Bond connectivity measured via relaxationassisted two-dimensional infrared spectroscopy. J. Chem.Phys 2008, 128 (10). 
64. Tesar SL; Kasyanenko VM; Rubtsov IV; Rubtsov GI; Burin AL, Theoretical Study of Internal Vibrational Relaxation and Energy Transport in Polyatomic Molecules. J. Phys. Chem. A 2013, 117 (2), 315-323. [PubMed: 23240557]

65. Rubtsova NI; Kurnosov AA; Burin AL; Rubtsov IV, Temperature Dependence of the Ballistic Energy Transport in Perfluoroalkanes. J. Phys. Chem. B 2014, 118 (28), 8381-8387. [PubMed: 24697782]

66. Levin DE; Schmitz AJ; Hines SM; Hines KJ; Tucker MJ; Brewer SH; Fenlon EE, Synthesis and evaluation of the sensitivity and vibrational lifetimes of thiocyanate and selenocyanate infrared reporters. RSC Adv. 2016, 6 (43), 36231-36237.

67. Gai XS; Coutifaris BA; Brewer SH; Fenlon EE, A direct comparison of azide and nitrile vibrational probes. Phys. Chem. Chem. Phys 2011, 13 (13), 5926-5930. [PubMed: 21336362]

68. Tucker MJ; Gai XS; Fenlon EE; Brewer SH; Hochstrasser RM, 2D IR photon echo of azido-probes for biomolecular dynamics. Phys. Chem. Chem. Phys 2011, 13 (6), 2237-2241. [PubMed: 21116553]

69. Bolje A; Urankar D; Kosmrlj J, Synthesis and NMR Analysis of 1,4-Disubstituted 1,2,3-Triazoles Tethered to Pyridine, Pyrimidine, and Pyrazine Rings. Eur. J. Org. Chem 2014, (36), 8167-8181.

70. Nicolaides A; Enyo T; Miura D; Tomioka H, p-phenylenecarbenonitrene and its halogen derivatives: How does resonance interaction between a nitrene and a carbene center affect the overall electronic configuration? J. Am. Chem. Soc 2001, 123 (11), 2628-2636. [PubMed: 11456932]

71. Chakraborty A; Dey S; Sawoo S; Adarsh NN; Sarkar A, Regioselective 1,3-Dipolar Cycloaddition Reaction of Azides with Alkoxy Alkynyl Fischer Carbene Complexes. Organometallics 2010, 29 (23), 6619-6622.

72. Pandey HD; Leitner DM, Thermalization and Thermal Transport in Molecules. J. Phys. Chem. Lett 2016, 7 (24), 5062-5067. [PubMed: 27973898]

73. Pandey HD; Leitner DM, Vibrational States and Nitrile Lifetimes of Cyanophenylalanine Isotopomers in Solution. J. Phys. Chem. A 2018, 122 (34), 6856-6863. [PubMed: 30091602]

74. Lipkin JS; Song R; Fenlon EE; Brewer SH, Modulating Accidental Fermi Resonance: What a Difference a Neutron Makes. J. Phys. Chem. Lett 2011, 2 (14), 1672-1676.

75. Zhang J; Wang L; Zhang J; Zhu JR; Pan X; Cui ZF; Wang JY; Fang WH; Li YL, Identifying and Modulating Accidental Fermi Resonance: 2D IR and DFT Study of 4-Azido-L-phenylalanine. J. Phys. Chem. B 2018, 122 (34), 8122-8133. [PubMed: 30067030]

76. Maj M; Ahn C; Kossowska D; Park K; Kwak K; Han H; Cho M, beta-Isocyanoalanine as an IR probe: comparison of vibrational dynamics between isonitrile and nitrile-derivatized IR probes. Phys. Chem. Chem. Phys 2015, 17 (17), 11770-11778. [PubMed: 25869854]

77. Coblentz Society I, Evaluated Infrared Reference Spectra In NIST Chemistry WebBook,NIST Standard Reference Database Number 69 [Online] Mallard P. J. L. a. W. G., Ed. National Institute of Standards and Technology: Gaithersburg, MD, 2018.

78. Varner C; Zhou X; Saxman ZK; Leger JD; Jayawickramarajah J; Rubtsov IV, Azido alkanes as convenient reporters for mobility within lipid membranes. Chem. Phys 2018, 512, 20-26.

79. Zhang WK; Markiewicz BN; Doerksen RS; Smith AB; Gai F, C equivalent to N stretching vibration of 5-cyanotryptophan as an infrared probe of protein local environment: what determines its frequency? Phys. Chem. Chem. Phys 2016, 18 (10), 7027-7034. [PubMed: 26343769]

80. Zheng JR; Kwak KW; Xie J; Fayer MD, Ultrafast carbon-carbon single-bond rotational isomerization in room-temperature solution. Science 2006, 313 (5795), 1951-1955. [PubMed: 17008529]

81. Rubtsov IV; Burin AL, Ballistic and diffusive vibrational energy transport in molecules. J. Chem.Phys 2019, 150 (2). 

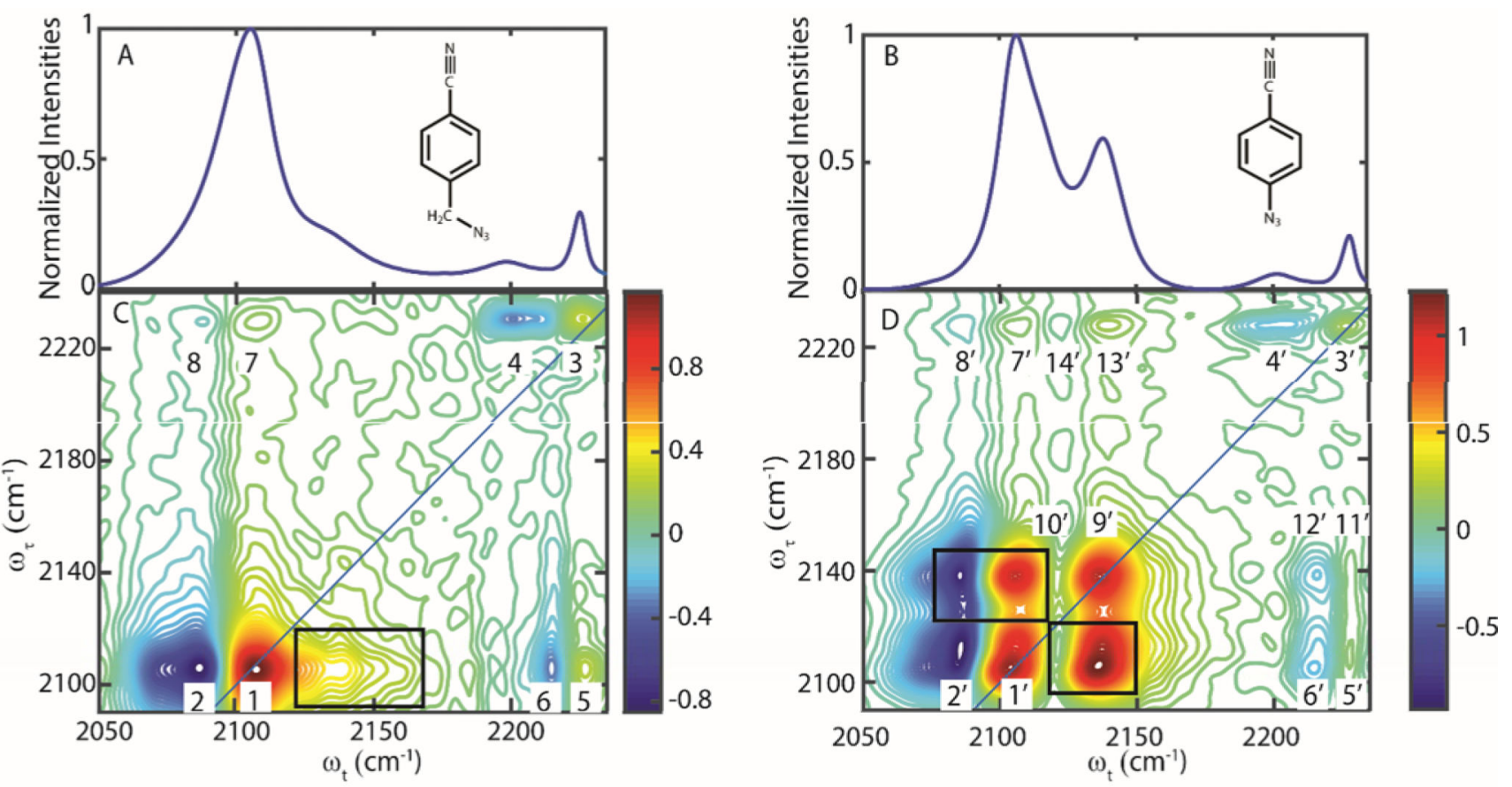

Figure 1.

FT IR spectra of A) PAMB and B) PAB in THF and absorptive 2D IR spectra of C) PAMB and D) PAB in in THF at $\mathrm{T}=3 \mathrm{ps}$. 


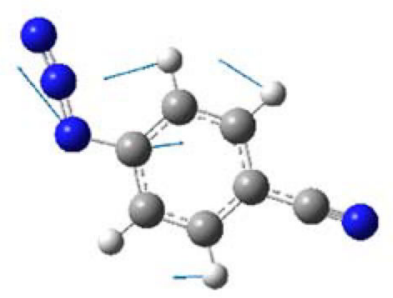

Ring+azido-

$1327 \mathrm{~cm}^{-1}$

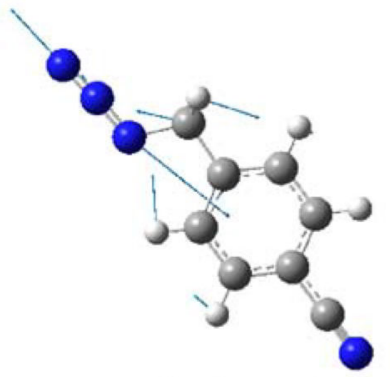

Methylene+ring+azido-

$1316 \mathrm{~cm}^{-1}$

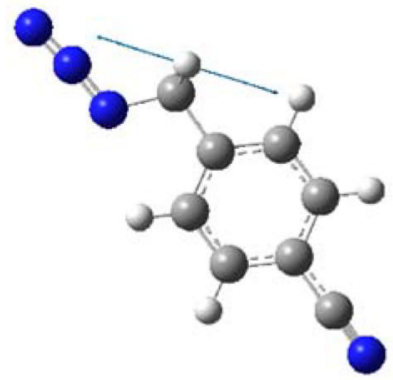

Methylene

$1217 \mathrm{~cm}^{-1}$

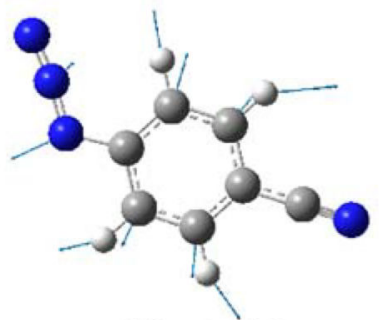

Ring+azido$839 \mathrm{~cm}^{-1}$

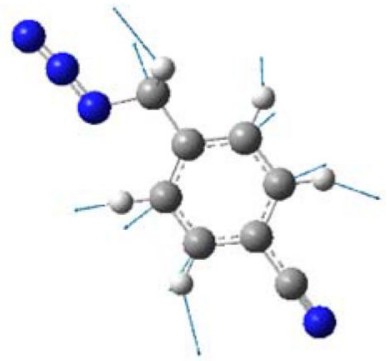

Ring+methylene $835 \mathrm{~cm}^{-1}$

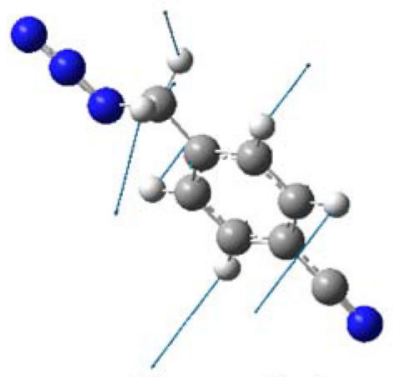

Ring+methylene $956 \mathrm{~cm}^{-1}$

Figure 2.

Vibrational transitions contributing to energy transfer pathway from the azido- to the cyanomode (top) PAB molecule and (middle and bottom) PAMB molecule. The phases and relative size of each arrow are accurate, but the absolute sizes of the arrows have been exaggerated to clearly distinguish the amplitude of the displacements of different atoms. 

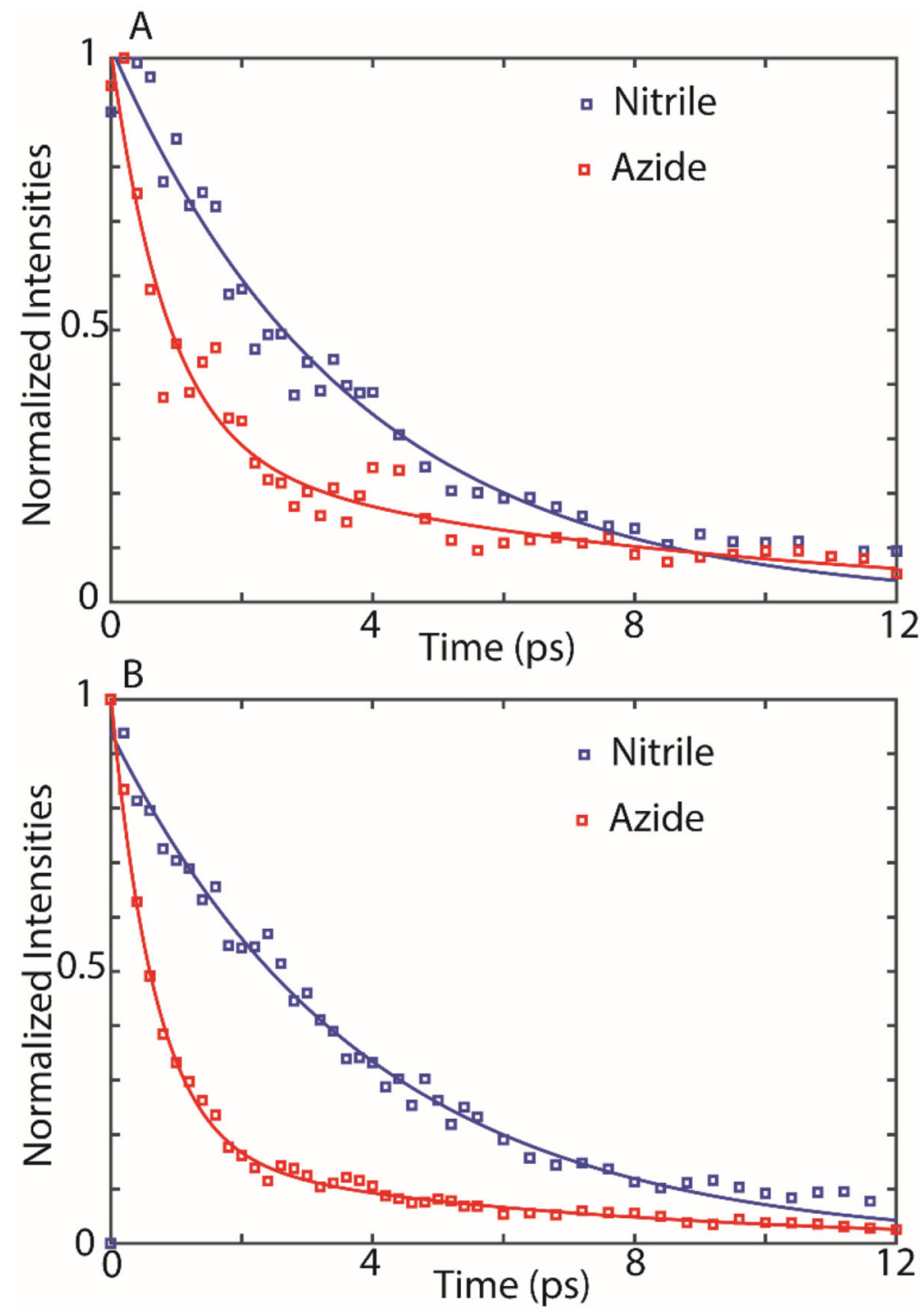

Figure 3.

Population decays of the cyano- and azido- transitions obtained from 2D IR spectrum for A) PAB and B) PAMB. The lines represent fits of the exponential decays of the azido- and cyano-. 


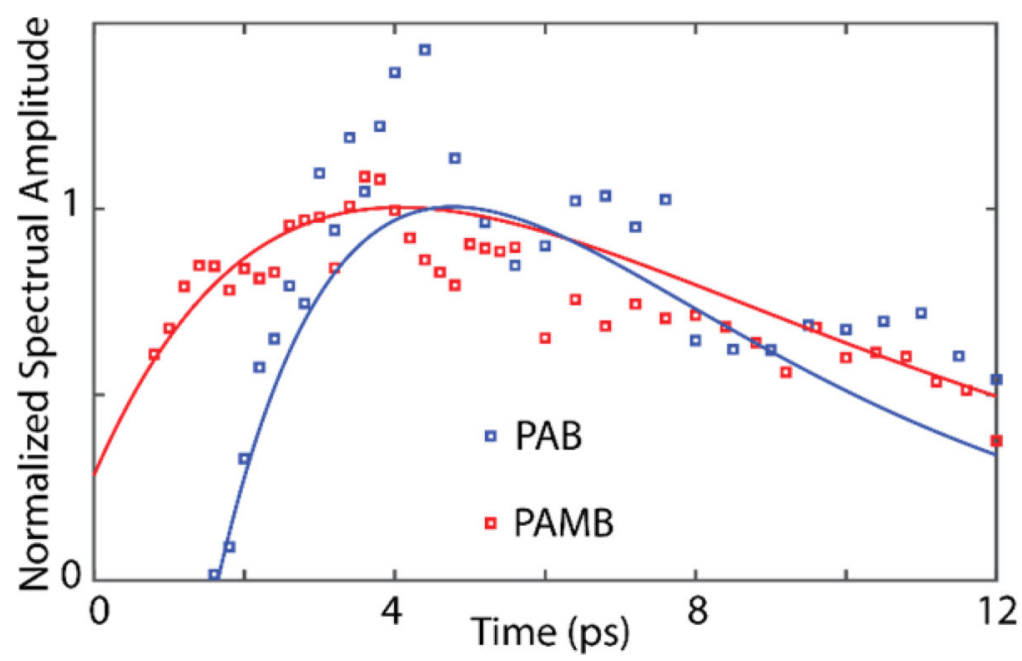

Figure 4.

2D IR cross peaks 5 and 5' of Figure 1 intensity growth and decay for PAMB (red) and PAB (blue). The data was fit to a bi-exponential growth and decay 

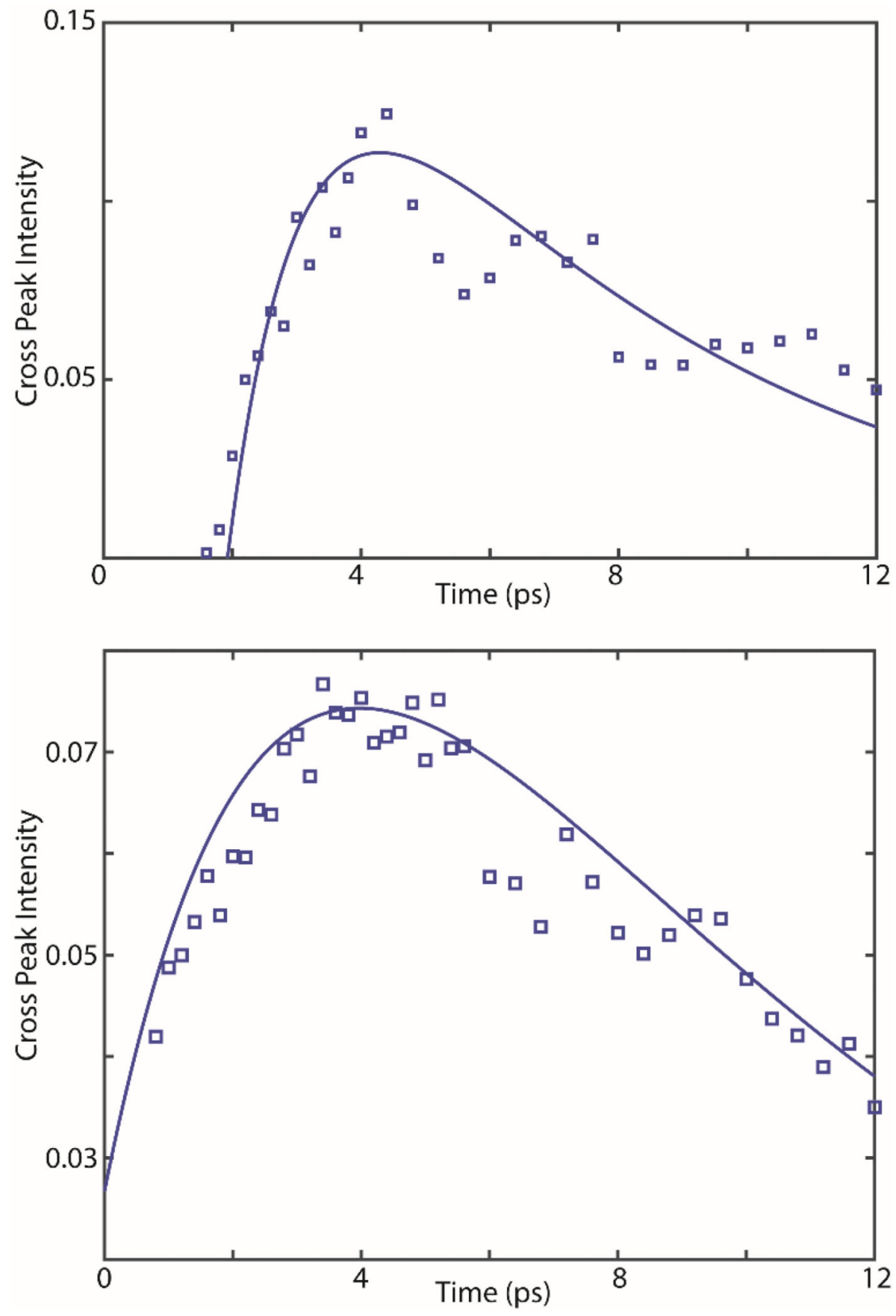

Figure 5.

Growth and decay of cross peak (top) 5' and (bottom) 5 from Figure 1 for PAB and PAMB compounds, respectively. The solid lines represent the fit to the derived kinetic model. 
Table 1.

Theoretically determined most probable combination bands giving rise to the Fermi resonance and contributing to the energy transfer pathway from the azido- to the cyano- mode. The third order coupling constant, $\Phi_{\mathrm{a} \beta \gamma}$, third order resonance parameter, $T F R_{a \beta \gamma}$, IVR rate, resonance width, $\Delta \omega$, and localized region are listed.

\begin{tabular}{|c|c|c|c|c|c|}
\hline \multicolumn{6}{|c|}{ PAB Azido- Calculated Frequency $\omega_{a}=2192 \mathrm{~cm}^{-1}$} \\
\hline $\begin{array}{l}\omega_{\beta}\left(\mathrm{cm}^{-1}\right) \\
\text { Localized Region }\end{array}$ & $\begin{array}{l}\omega_{\gamma}\left(\mathrm{cm}^{-1}\right) \\
\text { Localized Region }\end{array}$ & Contribution to IVR rate $\left(\mathrm{cm}^{-1}\right)$ & $\Phi_{a \beta \gamma}\left(\mathrm{cm}^{-1}\right)$ & $\Delta \omega\left(\mathrm{cm}^{-1}\right)$ & $T F R_{a \beta \gamma}$ \\
\hline $\begin{array}{l}1327 \\
\text { Ring+azido- }\end{array}$ & $\begin{array}{l}839 \\
\text { Ring+azido- }\end{array}$ & 13.82 & -49.87 & 26 & 1.913 \\
\hline \multicolumn{6}{|c|}{ PAMB Azido- Calculated Frequency $\omega_{a}=2169 \mathrm{~cm}^{-1}$} \\
\hline $\begin{array}{l}\omega_{\beta}\left(\mathrm{cm}^{-1}\right) \\
\text { Localized Region }\end{array}$ & $\begin{array}{l}\omega_{\gamma}\left(\mathrm{cm}^{-1}\right) \\
\text { Localized Region }\end{array}$ & Contribution to IVR rate $\left(\mathrm{cm}^{-1}\right)$ & $\Phi_{a \beta \gamma}\left(\mathrm{cm}^{-1}\right)$ & $\Delta \omega\left(\mathrm{cm}^{-1}\right)$ & $T F R_{a \beta \gamma}$ \\
\hline $\begin{array}{l}1316 \\
\text { Ring+methylene+azido- }\end{array}$ & $\begin{array}{l}835 \\
\text { Ring+methylene }\end{array}$ & 1.36 & 13.69 & 18 & 0.773 \\
\hline $\begin{array}{l}1217 \\
\text { Methylene }\end{array}$ & $\begin{array}{l}956 \\
\text { Ring+methylene }\end{array}$ & 0.29 & 3.98 & 4 & 0.943 \\
\hline
\end{tabular}

